\title{
BOUNDS ON THE MINIMUM DISTANCE OF ALGEBRAIC GEOMETRY CODES DEFINED OVER SOME FAMILIES OF SURFACES
}

\author{
YVES AUBRY, ELENA BERARDINI, FABIEN HERBAUT AND MARC PERRET
}

\author{
To the memory of Gilles Lachaud
}

\begin{abstract}
We prove lower bounds for the minimum distance of algebraic geometry codes over surfaces whose canonical divisor is either nef or antistrictly nef and over surfaces without irreducible curves of small genus. We sharpen these lower bounds for surfaces whose arithmetic Picard number equals one, surfaces without curves with small self-intersection and fibered surfaces. Finally we specify our bounds to the case of surfaces of degree $d \geq 3$ embedded in $\mathbb{P}^{3}$.
\end{abstract}

\section{CONTEnts}

1. Introduction 1

2. Background 3

3. The minimum distance of codes over some families of algebraic surfaces 8

4. Four improvements

5. An example: surfaces in $\mathbb{P}^{3} \quad 17$

$\begin{array}{lr}\text { References } & 19\end{array}$

\section{INTRODUCTION}

The construction of Goppa codes over algebraic curves ([8]) has enabled Tsfaman, Vlădut and Zink to beat the Gilbert-Varshamov bound ([19]). Since then, algebraic geometry codes over curves have been largely studied. Even though the same construction holds on varieties of higher dimension, the literature is less abundant in this context. However one can consult [15] for a survey of Little and [12] for an extensive use of intersection theory involving the Seshadri constant proposed by S. H. Hansen. Some work has also been undertaken in the direction of surfaces. Rational surfaces yielding to good codes were constructed by Couvreur in [7] from some blow-ups of the plane and by Blache et al. in [5] from Del Pezzo

Date: March 1, 2020.

2000 Mathematics Subject Classification. 14J99, 14G15, 14G50.

Key words and phrases. AG codes, algebraic surfaces, fibered surfaces, finite fields.

Funded by ANR grant ANR-15-CE39-0013-01 "Manta". 
surfaces. Codes from cubic surfaces where studied by Voloch and Zarzar in [20], from toric surfaces by J. P. Hansen in [11], from Hirzebruch surfaces by Nardi in [16], from ruled surfaces by one of the authors in [1] and from abelian surfaces by Haloui in [10] in the specific case of simple Jacobians of genus 2 curves, and by the authors in [4] for general abelian surfaces. Furthermore Voloch and Zarzar ([20], [21]) and Little and Schenck ([14]) have studied surfaces whose arithmetic Picard number is one.

The aim of this paper is to provide a study of the minimum distance $d(X, r H, S)$ of the algebraic geometry code $\mathcal{C}(X, r H, S)$ constructed from an algebraic surface $X$, a set $S$ of rational points on $X$, a rational effective ample divisor $H$ on $X$ avoiding $S$ and an integer $r>0$.

We prove in Section 3 lower bounds for the minimum distance $d(X, r H, S)$ under some specific assumptions on the geometry of the surface itself. Two quite wide families of surfaces are studied. The first one is that of surfaces whose canonical divisor is either nef or anti-strictly nef. The second one consists of surfaces which do not contain irreducible curves of low genus. We obtain the following theorem, where we denote, as in the whole paper, the finite field with $q$ elements by $\mathbb{F}_{q}$ and the virtual arithmetic genus of a divisor $D$ by $\pi_{D}$, and where we set $m:=\lfloor 2 \sqrt{q}\rfloor$.

Theorem. (Theorem 3.2 and Theorem 3.4) Let $X$ be an absolutely irreducible smooth projective algebraic surface defined over $\mathbb{F}_{q}$ whose canonical divisor is denoted by $K_{X}$. Consider a set $S$ of rational points on $X$, a rational effective ample divisor $H$ avoiding $S$, and a positive integer $r$. In order to compare the following bounds, we set

$$
d^{*}(X, r H, S):=\sharp S-r H^{2}(q+1+m)-m\left(\pi_{r H}-1\right) .
$$

1) (i) If $K_{X}$ is nef, then

$$
d(X, r H, S) \geq d^{*}(X, r H, S) .
$$

(ii) If $-K_{X}$ is strictly nef, then

$$
d(X, r H, S) \geq d^{*}(X, r H, S)+m r\left(\pi_{H}-1\right) .
$$

2) If there exists an integer $\ell>0$ such that any $\mathbb{F}_{q}$-irreducible curve lying on $X$ and defined over $\mathbb{F}_{q}$ has arithmetic genus strictly greater than $\ell$, then

$$
d(X, r H, S) \geq d^{*}(X, r H, S)+\left(r H^{2}-\frac{\pi_{r H}-1}{\ell}\right)(q+1+m) .
$$

Inside both families, adding some extra geometric assumptions on the surface yields in Section 4 to some improvements for these lower bounds. This is the case for surfaces whose arithmetic Picard number is one, for surfaces without irreducible curves defined over $\mathbb{F}_{q}$ with small self-intersection, so as for fibered surfaces. Theorems 4.8 and 4.9 (that hold for fibered surfaces) improve the bounds 
of Theorems 3.2 and 3.4 (that hold for the whole wide families). Indeed the bound on the minimum distance $d(X, r H, S)$ is increased by the non-negative defect $\delta(B)=q+1+m g_{B}-\sharp B\left(\mathbb{F}_{q}\right)$ of the base curve $B$. Finally in Section 5 we specify our bounds to the case of surfaces of degree $d \geq 3$ embedded in $\mathbb{P}^{3}$.

Characterizing surfaces that yield good codes seems to be a complex question. It is not the goal of our paper to produce good codes: we aim to give theoretical bounds on the minimum distance of algebraic geometry codes on general surfaces. However one can derive from our work one or two heuristics. Indeed, Theorem 3.4 suggests to look for surfaces with no curves of small genus and fibered surfaces provide natural examples of such surfaces (see Theorem 4.9).

\section{BACKGROUND}

Codes from algebraic surfaces are defined in the same way as on algebraic curves: we evaluate some functions with prescribed poles on some sets of rational points. Whereas the key tool for the study of the minimum distance in the 1dimensional case is the mere fact that a function has as many zeroes as poles, in the 2-dimensional case most of the proofs rest on intersection theory.

We sum up in this section the few results on intersection theory we need. Following the authors cited in the Introduction we recall the definition of the algebraic geometry codes. We also recall quickly how the dimension of the code can be bounded from below under the assumption of the injectivity of the evaluation map. Then we prove a lemma that will be used in the course of the paper to bound from below the minimum distance of the code for several families of surfaces. Finally, we recall some results on the number of rational points on curves over finite fields.

2.1. Intersection theory. Intersection theory has almost become a mainstream tool to study codes over surfaces (see [1], [12], [20], [21], [14], [4]) and it is also central in our proofs. We do not recall here the classical definitions of the different equivalent classes of divisors and we refer the reader to $[13, \S \mathrm{V}]$ for a presentation. We denote by $\operatorname{NS}(X)$ the arithmetic Néron-Severi group of a smooth surface $X$ defined over $\mathbb{F}_{q}$ whose rank is called the arithmetic Picard number of $X$, or Picard number for short. Recall that a divisor $D$ on $X$ is said to be nef (respectively strictly nef) if D.C $\geq 0$ (respectively $D . C>0$ ) for any irreducible curve $C$ on $X$. A divisor $D$ is said to be anti-ample if $-D$ is ample, anti-nef if $-D$ is nef and anti-strictly nef if $-D$ is strictly nef. Let us emphasize three classical results we will use in this paper.

The first one is (a generalisation of) the adjunction formula (see $[13, \S \mathrm{V}, \mathrm{Ex}-$ ercise 1.3]). For any $\mathbb{F}_{q}$-irreducible curve $D$ on $X$ of arithmetic genus $\pi_{D}$, we have

$$
D \cdot\left(D+K_{X}\right)=2 \pi_{D}-2
$$


where $K_{X}$ is the canonical divisor on $X$. This formula allows us to define the virtual arithmetic genus of any divisor $D$ on $X$.

The second one is the corollary of the Hodge index theorem stating that if $H$ and $D$ are two divisors on $X$ with $H$ ample, then

$$
H^{2} D^{2} \leq(H . D)^{2},
$$

where equality holds if and only if $H$ and $D$ are numerically proportional.

The last one is a simple outcome of Bézout's theorem in projective spaces (and the trivial part of the Nakai-Moishezon criterion). It ensures that for any ample divisor $H$ on $X$ and for any irreducible curve $C$ on $X$, we have $H^{2}>0$ and H.C $>0$.

\subsection{Algebraic geometry codes.}

2.2.1. Definition of $A G$ codes. We study, as in the non-exhaustive list of papers [1], [20], [7], [12], [21], [10], [14] and [4], the generalisation of Goppa algebraic geometry codes from curves to surfaces. In the whole paper we consider an absolutely irreducible smooth projective algebraic surface $X$ defined over $\mathbb{F}_{q}$ and a set $S$ of rational points on $X$. Given a rational effective ample divisor $G$ on $X$ avoiding $S$, the algebraic geometry code, or AG code for short, is defined by evaluating the elements of the Riemann-Roch space $L(G)$ at the points of $S$. Precisely we define the linear code $\mathcal{C}(X, G, S)$ as the image of the evaluation map ev : $L(G) \longrightarrow \mathbb{F}_{q}^{\sharp S}$.

2.2.2. Length and dimension of $A G$ codes. From the very definition, the length of the code is $\sharp S$. As soon as the morphism ev is injective - see (7) for a sufficient condition - the dimension of the code equals $\ell(G)=\operatorname{dim}_{\mathbb{F}_{q}} L(G)$ which can be easily bounded from below using standard algebraic geometry tools as follows. By Riemann-Roch theorem (see $[13, \mathrm{~V}, \S 1]$ ), we have

$$
\ell(G)-s(G)+\ell\left(K_{X}-G\right)=\frac{1}{2} G \cdot\left(G-K_{X}\right)+1+p_{a}(X)
$$

where $p_{a}(X)$ is the arithmetic genus of $X$, and where the so-called superabundance $s(G)$ of $G$ in $X$ is non-negative (as it is the dimension of some vector space). Now, under the assumption that

$$
K_{X} \cdot A<G . A,
$$

for some ample divisor $A$, we have from [13, V, Lemma 1.7] that $\ell\left(K_{X}-G\right)=0$. Thus, if the evaluation map ev is injective and under assumption (3), we get the lower bound

$$
\operatorname{dim} \mathcal{C}(X, G, S)=\ell(G) \geq \frac{1}{2} G \cdot\left(G-K_{X}\right)+1+p_{a}(X)
$$

for the dimension of the code $\mathcal{C}(X, G, S)$. 
2.2.3. Toward the minimum distance of $A G$ codes. It follows that the difficulty lies in the estimation of the minimum distance $d(X, G, S)$ of the code. For any non-zero $f \in L(G)$, we introduce the number $N(f)$ of rational points of the divisor of zeroes of $f$. The Hamming weight $w(\mathrm{ev}(f))$ of the codeword ev $(f)$ satisfies

$$
w(\mathrm{ev}(f)) \geq \sharp S-N(f),
$$

from which it follows that

$$
d(X, G, S) \geq \sharp S-\max _{f \in L(G) \backslash\{0\}} N(f) .
$$

We also deduce from (5) that

$$
\text { ev is injective if } \max _{f \in L(G) \backslash\{0\}} N(f)<\sharp S \text {. }
$$

We now broadly follow the way of [10]. We associate to any non-zero function $f \in L(G)$ the rational effective divisor

$$
D_{f}:=G+(f)=\sum_{i=1}^{k} n_{i} D_{i} \geq 0,
$$

where $(f)$ is the principal divisor defined by $f$, the $n_{i}$ are positive integers and each $D_{i}$ is a reduced $\mathbb{F}_{q}$-irreducible curve.

Note that in this setting, the integer $k$ and the curves $D_{i}$ 's depend on $f \in L(G)$. Several lower bounds for the minimum distance $d(X, G, S)$ in this paper will follow from the key lemma below.

Lemma 2.1. Let $X$ be a smooth projective surface defined over $\mathbb{F}_{q}$, let $S$ be a set of rational points on $X$ and let $G$ be a rational effective divisor on $X$ avoiding S. Set $m=\lfloor 2 \sqrt{q}\rfloor$ and keep the notations introduced in (8). If there exist nonnegative real numbers $a, b_{1}, b_{2}, c$, such that for any non-zero $f \in L(G)$ the three following assumptions are satisfied

(1) $k \leq a$,

(2) $\sum_{i=1}^{k} \pi_{D_{i}} \leq b_{1}+k b_{2}$ and

(3) for any $1 \leq i \leq k$ we have $\sharp D_{i}\left(\mathbb{F}_{q}\right) \leq c+m \pi_{D_{i}}$

then the minimum distance $d(X, G, S)$ of $\mathcal{C}(X, G, S)$ satisfies

$$
d(X, G, S) \geq \sharp S-a\left(c+m b_{2}\right)-m b_{1} .
$$

Proof. Let us write the principal divisor $(f)=(f)_{0}-(f)_{\infty}$ as the difference of its effective divisor of zeroes minus its effective divisor of poles. Since $G$ is effective and $f$ belongs to $L(G)$, we have $(f)_{\infty} \leq G$. Hence, formula (8) reads $G+(f)_{0}-(f)_{\infty}=\sum_{i=1}^{k} n_{i} D_{i}$, that is

$$
(f)_{0}=\sum_{i=1}^{k} n_{i} D_{i}+(f)_{\infty}-G \leq \sum_{i=1}^{k} n_{i} D_{i} .
$$


This means that any $\mathbb{F}_{q}$-rational point of $(f)_{0}$ lies in some $D_{i}$ so

$$
N(f) \leq \sum_{i=1}^{k} \sharp D_{i}\left(\mathbb{F}_{q}\right) .
$$

Then it follows successively from the assumptions of the lemma that

$$
N(f) \leq \sum_{i=1}^{k}\left(c+m \pi_{D_{i}}\right) \leq k c+m\left(b_{1}+k b_{2}\right) \leq m b_{1}+a\left(c+m b_{2}\right) .
$$

Finally Lemma 2.1 follows from (6).

Remark 2.2. In several papers, the point of departure to estimate the minimum distance is a bound on the number of components $k$, which corresponds to the condition (1) of Lemma 2.1 above. In the special case where $\operatorname{NS}(S)=\mathbb{Z} H$ and $G=r H$, for $H$ an ample divisor on $X$, Voloch and Zarzar have proven in [20] that $k \leq r$. In the present paper we obtain a bound on $k$ in a more general context, that is when the Néron-Severi group has rank greater than one (see for example Lemma 3.1, point (1) of Lemma 3.3 and point (1) of Lemma 4.5).

2.3. Two upper bounds for the number of rational points on curves. We manage to fulfill assumption (3) in Lemma 2.1 using the bounds on the number of rational points given in Theorem 2.3 and Proposition 2.4 below. Point (2) of Theorem 2.3 appears in the proof of Theorem 3.3 of Little and Schenck in [14] within a more restrictive context, whereas point (1) follows from [2]. We state a general theorem and give here the full proof for the sake of completeness following [14].

Theorem 2.3 (Aubry-Perret [2] and Little-Schenck [14]). Let D be an $\mathbb{F}_{q}$-irreducible curve of arithmetic genus $\pi_{D}$ lying on a smooth projective algebraic surface. Then,

(1) we have $\sharp D\left(\mathbb{F}_{q}\right) \leq q+1+m \pi_{D}$.

(2) (Little-Schenck) If moreover $D$ is not absolutely irreducible, we have

$$
\sharp D\left(\mathbb{F}_{q}\right) \leq \pi_{D}+1 \text {. }
$$

Proof. We first prove the second item, following the proof of [14, Th. 3.3]. Since $D$ is $\mathbb{F}_{q}$-irreducible, the Galois group $\operatorname{Gal}\left(\overline{\mathbb{F}}_{q} / \mathbb{F}_{q}\right)$ acts transitively on the set of its $\bar{r} \geq 1$ absolutely irreducible components $D_{1}, \ldots, D_{\bar{r}}$. Since a $\mathbb{F}_{q}$-rational point on $D$ is stable under the action of $\operatorname{Gal}\left(\overline{\mathbb{F}}_{q} / \mathbb{F}_{q}\right)$, it lies in the intersection $\cap_{1 \leq i \leq \bar{r}} D_{i}$. Under the assumption that $D$ is not absolutely irreducible, that is $\bar{r} \geq 2$, it follows that $\sharp D\left(\mathbb{F}_{q}\right) \leq \sharp\left(D_{i} \cap D_{j}\right)\left(\overline{\mathbb{F}}_{q}\right) \leq D_{i} . D_{j}$ for every couple $(i, j)$ with $i \neq j$.

As a divisor, $D$ can be written over $\overline{\mathbb{F}}_{q}$ as $D=\sum_{i=1}^{\bar{r}} a_{i} D_{i}$. By transitivity of the Galois action, we have $a_{1}=\cdots=a_{\bar{r}}=a$. Now since $D$ can be assumed to be reduced, we have $a=1$, so that finally $D=\sum_{i=1}^{\bar{r}} D_{i}$. Using the adjonction formula (1) for $D$ and each $D_{i}$, and taking into account that $\pi_{D_{i}} \geq 0$ for any $i$, 
we get

$$
\begin{aligned}
2 \pi_{D}-2 & =\left(K_{X}+D\right) \cdot D \\
& =\sum_{i=1}^{\bar{r}}\left(K_{X}+D_{i}\right) \cdot D_{i}+\sum_{i \neq j} D_{i} \cdot D_{j} \\
& =\sum_{i=1}^{\bar{r}}\left(2 \pi_{D_{i}}-2\right)+\sum_{i \neq j} D_{i} \cdot D_{j} \\
& \geq-2 \bar{r}+\sum_{i \neq j} D_{i} \cdot D_{j} .
\end{aligned}
$$

Since there are $\bar{r}(\bar{r}-1)$ pairs $(i, j)$ with $i \neq j$, we deduce that for at least one such pair $\left(i_{0}, j_{0}\right)$, we have

$$
D_{i_{0}} \cdot D_{j_{0}} \leq \frac{2\left(\pi_{D}-1+\bar{r}\right)}{\bar{r}(\bar{r}-1)}
$$

It is then easily checked that the left hand side of the former inequality is a decreasing function of $\bar{r} \geq 2$, so that we obtain

$$
\sharp D\left(\mathbb{F}_{q}\right) \leq D_{i_{0}} \cdot D_{j_{0}} \leq \frac{2\left(\pi_{D}-1+2\right)}{2(2-1)}=\pi_{D}+1
$$

and the second item is proved.

The first item follows from Aubry-Perret's bound in [2] in case $D$ is absolutely irreducible, that is in case $\bar{r}=1$, and from the second item in case $D$ is not absolutely irreducible since $\pi_{D}+1 \leq q+1+m \pi_{D}$.

The following bound will be usefull in Subsection 4.3 for the study of codes from fibered surfaces.

Proposition 2.4 (Aubry-Perret [3]). Let $C$ be a smooth projective absolutely irreducible curve of genus $g_{C}$ over $\mathbb{F}_{q}$ and $D$ be an $\mathbb{F}_{q}$-irreducible curve having $\bar{r}$ absolutely irreducible components $\bar{D}_{1}, \ldots, \bar{D}_{\bar{r}}$. Suppose there exists a regular map $D \rightarrow C$ in which none absolutely irreducible component maps onto a point. Then

$$
\left|\sharp D\left(\mathbb{F}_{q}\right)-\sharp C\left(\mathbb{F}_{q}\right)\right| \leq(\bar{r}-1) q+m\left(\pi_{D}-g_{C}\right) .
$$

Proof. Since $C$ is smooth and none geometric component of $D$ maps onto a point, the map $D \rightarrow C$ is flat. Hence by [3, Th.14] we have

$$
\left|\sharp D\left(\mathbb{F}_{q}\right)-\sharp C\left(\mathbb{F}_{q}\right)\right| \leq(\bar{r}-1)(q-1)+m\left(\sum_{i=1}^{\bar{r}} g_{\bar{D}_{i}}-g_{C}\right)+\Delta_{D}
$$

where $\Delta_{D}=\sharp \tilde{D}\left(\overline{\mathbb{F}}_{q}\right)-\sharp D\left(\overline{\mathbb{F}}_{q}\right)$ with $\tilde{D}$ the normalization of $D$. The result follows from [3, Lemma 2] where it is proved that $m \sum_{i=1}^{\bar{r}} g_{\bar{D}_{i}}+\Delta_{D}-\bar{r}+1 \leq m \pi_{D}$. 


\section{The minimum distance of Codes over some families of Algebraic SURFACES}

We are unfortunately unable to fulfill simultaneously assumptions (1) and (2) of Lemma 2.1 for general surfaces. So we focus on two families of algebraic surfaces where we do succeed. To begin with, let us fix some common notations.

We consider a rational effective ample divisor $H$ on the surface $X$ avoiding a set $S$ of rational points on $X$ and for a positive integer $r$ we consider $G=r H$. We study, in accordance to Section 2.2, the evaluation code $\mathcal{C}(X, r H, S)$ and we denote by $d(X, r H, S)$ its minimum distance.

\subsection{Surfaces whose canonical divisor is either nef or anti-strictly nef.}

We study in this section codes defined over surfaces such that either the canonical divisor $K_{X}$ is nef, or its opposite $-K_{X}$ is strictly nef. This family is quite large. It contains, for instance:

- surfaces whose canonical divisor $K_{X}$ is anti-ample.

- Minimal surfaces of Kodaira dimension 0, for which the canonical divisor is numerically zero, hence nef. These are abelian surfaces, $K 3$ surfaces, Enriques surfaces and hyperelliptic or quasi-hyperelliptic surfaces (see [6]).

- Minimal surfaces of Kodaira dimension 2. These are the so called minimal surfaces of general type. For instance, surfaces in $\mathbb{P}^{3}$ of degree $d \geq 4$, without curves $C$ with $C^{2}=-1$, are minimal of general type.

- Surfaces whose arithmetic Picard number is one.

- Surfaces of degree 3 embedded in $\mathbb{P}^{3}$.

The main theorem of this section (Theorem 3.2) rests mainly on the next lemma designed to fulfill assumptions (1) and (2) of Lemma 2.1.

Lemma 3.1. Let $D=\sum_{i=1}^{k} n_{i} D_{i}$ be the decomposition as a sum of $\mathbb{F}_{q}$-irreducible and reduced curves of an effective divisor $D$ linearly equivalent to $r H$. Then we have:

(1) $k \leq r H^{2}$

(2) (i) if $K_{X}$ is nef, then $\sum_{i=1}^{k} \pi_{D_{i}} \leq \pi_{r H}-1+k$;

(ii) if $-K_{X}$ is strictly nef, then $\sum_{i=1}^{k} \pi_{D_{i}} \leq \pi_{r H}-1-\frac{1}{2} r H \cdot K_{X}+\frac{1}{2} k$.

Proof. Using that $D$ is numerically equivalent to $r H$, that $n_{i}>0$ and $D_{i} . H>0$ for every $i=1, \ldots, k$ since $H$ is ample, we prove item (1):

$$
r H . H=D . H=\sum_{i=1}^{k} n_{i} D_{i} . H \geq \sum_{i=1}^{k} D_{i} \cdot H \geq k .
$$

Now we apply inequality (2) to $H$ and $D_{i}$ for every $i$, to get $D_{i}^{2} H^{2} \leq\left(D_{i} \cdot H\right)^{2}$. We thus have, together with adjunction formula (1) and inequality $H^{2}>0$,

$$
\pi_{D_{i}}-1 \leq\left(D_{i} . H\right)^{2} / 2 H^{2}+D_{i} \cdot K_{X} / 2 .
$$


To prove point (i) of item (2) we sum from $i=1$ to $k$ and thus obtain

$$
\begin{aligned}
\sum_{i=1}^{k} \pi_{D_{i}}-k & \leq \frac{1}{2 H^{2}} \sum_{i=1}^{k}\left(D_{i} \cdot H\right)^{2}+\frac{1}{2} \sum_{i=1}^{k} D_{i} \cdot K_{X} \\
& \leq \frac{1}{2 H^{2}}\left(\sum_{i=1}^{k} n_{i} D_{i} \cdot H\right)^{2}+\frac{1}{2} \sum_{i=1}^{k} n_{i} D_{i} \cdot K_{X} \\
& \leq \frac{(r H \cdot H)^{2}}{2 H^{2}}+\frac{r H \cdot K_{X}}{2} \\
& =\pi_{r H}-1,
\end{aligned}
$$

where we use the positivity of the coefficients $n_{i}$, the numeric equivalence between $D$ and $\sum_{i=1}^{k} n_{i} D_{i}$, the fact that $H$ is ample and the hypothesis taken on $K_{X}$.

Under the hypothesis of point (ii) we have $D_{i} \cdot K_{X} \leq-1$. Replacing in the first line of (11) gives $\sum_{i=1}^{k} \pi_{D_{i}}-k \leq \frac{1}{2 H^{2}} \sum_{i=1}^{k}\left(D_{i} \cdot H\right)^{2}-\frac{k}{2}$. We conclude in the same way.

Theorem 3.2. Let $H$ be a rational effective ample divisor on a surface $X$ avoiding a set $S$ of rational points and let $r$ be a positive integer. We set

$$
d^{*}(X, r H, S):=\sharp S-r H^{2}(q+1+m)-m\left(\pi_{r H}-1\right) .
$$

(i) If $K_{X}$ is nef, then

$$
d(X, r H, S) \geq d^{*}(X, r H, S) .
$$

(ii) If $-K_{X}$ is strictly nef, then

$$
d(X, r H, S) \geq d^{*}(X, r H, S)+m r\left(\pi_{H}-1\right) .
$$

Proof. The theorem follows from Lemma 2.1 for which assumptions (1) and (2) hold from Lemma 3.1 and assumption (3) holds from Theorem 2.3.

3.2. Surfaces without irreducible curves of small genus. We consider in this section surfaces $X$ with the property that there exists an integer $\ell \geq 1$ such that any $\mathbb{F}_{q}$-irreducible curve $D$ lying on $X$ and defined over $\mathbb{F}_{q}$ has arithmetic genus $\pi_{D} \geq \ell+1$. It turns out that under this hypothesis, we can fulfill assumptions (1) and (2) of Lemma 2.1 without any hypothesis on $K_{X}$ contrary to the setting of Section 3.1.

Examples of surfaces with this property do exist. For instance:

- simple abelian surfaces satisfy this property for $\ell=1$ (see [4] for abelian surfaces with this property for $\ell=2$ ).

- Fibered surfaces on a smooth base curve $B$ of genus $g_{B} \geq 1$ and generic fiber of arithmetic genus $\pi_{0} \geq 1$, and whose singular fibers are $\mathbb{F}_{q}$-irreducible, do satisfy this property for $\ell=\min \left(g_{B}, \pi_{0}\right)-1$. 
- Smooth surfaces in $\mathbb{P}^{3}$ of degree $d$ whose arithmetic Picard group is generated by the class of an hyperplane section do satisfy this property for $\ell=\frac{(d-1)(d-2)}{2}-1$ (see Lemma 5.2).

Lemma 3.3. Let $X$ be a surface without $\mathbb{F}_{q}$-irreducible curves of arithmetic genus less than or equal to $\ell$ for $\ell$ a positive integer. Consider a rational effective ample divisor $H$ on $X$ and a positive integer $r$. Let $D=\sum_{i=1}^{k} n_{i} D_{i}$ be the decomposition as a sum of $\mathbb{F}_{q}$-irreducible and reduced curves of an effective divisor $D$ linearly equivalent to $r H$. Then we have

(1) $k \leq \frac{\pi_{r H}-1}{\ell}$;

(2) $\sum_{i=1}^{k} \pi_{D_{i}} \leq \pi_{r H}-1+k$.

In case $X$ falls in both families of Section 3.1 and of this Section 3.2, the present new bound of the first item for $k$ is better than the one of Lemma 3.1 if and only if $\pi_{r H}-1<\ell r H^{2}$, that is if and only if $\ell>\frac{H \cdot K_{X}}{2 H^{2}}+\frac{r}{2}$. In the general setting, this inequality sometimes holds true, sometimes not. As a matter of example, supposed $K_{X}$ to be ample and let us consider $H=K_{X}$. In this setting the inequality holds if and only if $r<2 \ell-1$.

Proof. By assumption, we have $0 \leq \ell \leq \pi_{D_{i}}-1$ and $n_{i} \geq 1$ for any $1 \leq i \leq k$, hence using adjunction formula (1), we have

$$
2 \ell k \leq 2 \sum_{i=1}^{k}\left(\pi_{D_{i}}-1\right) \leq 2 \sum_{i=1}^{k} n_{i}\left(\pi_{D_{i}}-1\right)=\sum_{i=1}^{k} n_{i} D_{i}^{2}+\sum_{i=1}^{k} n_{i} D_{i} \cdot K_{X} .
$$

Moreover using (2) and (8), we get

$$
2 \ell k \leq \sum_{i=1}^{k} n_{i} \frac{\left(D_{i} \cdot H\right)^{2}}{H^{2}}+\left(\sum_{i=1}^{k} n_{i} D_{i}\right) \cdot K_{X} \leq \sum_{i=1}^{k} n_{i}^{2} \frac{\left(D_{i} \cdot H\right)^{2}}{H^{2}}+r H . K_{X} .
$$

Since $H$ is ample, we obtain

$$
2 \ell k \leq \sum_{i, j=1}^{k} n_{i} n_{j} \frac{\left(D_{i} \cdot H\right)\left(D_{j} \cdot H\right)}{H^{2}}+r H \cdot K_{X}=\frac{\left(\sum_{i,=1}^{k} n_{i} D_{i} \cdot H\right)^{2}}{H^{2}}+r H \cdot K_{X} .
$$

By (8), we conclude that

$$
2 \ell k \leq \frac{(r H . H)^{2}}{H^{2}}+r H \cdot K_{X}=2\left(\pi_{r H}-1\right),
$$

and both items of Lemma 3.3 follow.

Theorem 3.4. Let $X$ be a surface without $\mathbb{F}_{q}$-irreducible curves of arithmetic genus less than or equal to $\ell$ for $\ell$ a positive integer. Consider a rational effective ample divisor $H$ on $X$ avoiding a finite set $S$ of rational points and let $r$ be a positive integer. Then we have

$$
d(X, r H, S) \geq d^{*}(X, r H, S)+\left(r H^{2}-\frac{\pi_{r H}-1}{\ell}\right)(q+1+m) .
$$


Proof. The theorem follows from Lemma 2.1, for which items (1) and (2) hold from Lemma 3.3 and item (3) holds from Theorem 2.3.

\section{Four IMPROVEMENTS}

In this section we manage to obtain better parameters for conditions (1), (2) or (3) of Lemma 2.1 in four cases: for surfaces of arithmetic Picard number one, for surfaces which do not contain $\mathbb{F}_{q}$-irreducible curves of small self-intersection and whose canonical divisor is either nef or anti-nef, for fibered surfaces with nef canonical divisor, and for fibered surfaces whose singular fibers are $\mathbb{F}_{q}$-irreducible curves.

4.1. Surfaces with Picard number one. As mentioned in the Introduction, the case of surfaces $X$ whose arithmetic Picard number equals one has already attracted some interest (see [21], [20], [14] and [5]). We prove in this subsection Lemma 4.1 and Theorem 4.3 which improve, under this rank one assumption, the bounds of Lemma 3.1 and Theorem 3.2. These new bounds depend on the sign of $3 H^{2}+H . K_{X}$, where $H$ is the ample generator of $\mathrm{NS}(X)$.

Lemma 4.1. Let $X$ be a smooth projective surface of arithmetic Picard number one. Let $H$ be the ample generator of $\mathrm{NS}(X)$ and let $r$ be a positive integer. For any non-zero function $f \in L(r H)$ consider the decomposition $D_{f}=\sum_{i=1}^{k} n_{i} D_{i}$ into $\mathbb{F}_{q}$-irreducible and reduced curves $D_{i}$ with positive integer coefficients $n_{i}$ as in (8). Then the sum of the arithmetic genera of the curves $D_{i}$ satisfies:

(i) $\sum_{i=1}^{k} \pi_{D_{i}} \leq(k-1) \pi_{H}+\pi_{(r-k+1) H}$ if $3 H^{2}+H \cdot K_{X} \geq 0$;

(ii) $\sum_{i=1}^{k} \pi_{D_{i}} \leq H^{2}(r-k)^{2} / 2+H^{2}(r-2 k)+k$ if $3 H^{2}+H . K_{X}<0$.

Remark 4.2. Note that the condition $3 H^{2}+H \cdot K_{X} \geq 0$ is satisfied as soon as $H . K_{X} \geq 0$. It is also satisfied in the special case where $K_{X}=-H$ which corresponds to Del Pezzo surfaces.

Proof. In order to prove the first item, we consider a non-zero function $f \in L(r H)$ and we keep the notation already introduced in (8), namely $D_{f}=\sum_{i=1}^{k} n_{i} D_{i}$. As $\mathrm{NS}(X)=\mathbb{Z} H$, for all $i$ we have $D_{i}=a_{i} H$ and we know by Lemma 2.2 in [21] that $k \leq r$. Intersecting with the ample divisor $H$ enables to prove that for all $i$ we have $a_{i} \geq 1$ and that $\sum_{i=1}^{k} n_{i} a_{i}=r$. Thus to get an upper bound for $\sum_{i=1}^{k} \pi_{D_{i}}=$ $\sum_{i=1}^{k} \pi_{a_{i} H}$, we are reduced to bounding $\left(\sum_{i=1}^{k} a_{i}^{2}\right) H^{2} / 2+\left(\sum_{i=1}^{k} a_{i}\right) H . K_{X} / 2+k$ under the constraint $\sum_{i=1}^{k} a_{i} n_{i}=r$. Our strategy is based on the two following arguments.

First, the condition $3 H^{2}+H . K_{X} \geq 0$ guarantees that $a \mapsto \pi_{a H}$ is an increasing sequence. Indeed, for integers $a^{\prime}>a \geq 1$ we have $\pi_{a^{\prime} H} \geq \pi_{a H}$ if and only if $\left(a+a^{\prime}\right) H^{2} \geq-H . K_{X}$, which is true under the condition above because $a+a^{\prime} \geq 3$. As a consequence, if we fix an index $i$ between 1 and $k$ and if we consider that 
the product $n_{i} a_{i}$ is constant, then the value of $\pi_{a_{i} H}$ is maximum when $a_{i}$ is, that is when $a_{i}=n_{i} a_{i}$ and $n_{i}=1$.

Secondly, assume that all the $n_{i}$ equal 1 and that $\sum_{i=1}^{k} a_{i}=r$. We are now reduced to bounding $\sum_{i=1}^{k} a_{i}^{2}$. We can prove that the maximum is reached when all the $a_{i}$ equal 1 except one which equals $r-k+1$. Otherwise, suppose for example that $2 \leq a_{1} \leq a_{2}$. Then $a_{1}^{2}+a_{2}^{2}<\left(a_{1}-1\right)^{2}+\left(a_{2}+1\right)^{2}$ and $\sum_{i=1}^{k} a_{i}^{2}$ is not maximum, and the first item is thus proved.

For the second item, using the adjonction formula we get

$$
\sum_{i=1}^{k} \pi_{D_{i}}-k \leq \frac{1}{2 H^{2}} \sum_{i=1}^{k}\left(D_{i} \cdot H\right)^{2}+\frac{1}{2} \sum_{i=1}^{k} D_{i} \cdot K_{X} .
$$

Again as $\operatorname{NS}(X)=\mathbb{Z} H$, for all $i$ we have $D_{i}=a_{i} H$. Thus we get

$$
\sum_{i=1}^{k} \pi_{D_{i}}-k \leq \frac{1}{2 H^{2}} \sum_{i=1}^{k} a_{i}^{2}\left(H^{2}\right)^{2}+\frac{1}{2} \sum_{i=1}^{k} a_{i} H . K_{X} .
$$

Now using that $H \cdot K_{X} \leq-3 H^{2}$ by hypothesis, that $\sum_{i=1}^{k} a_{i} \geq k$ since every $a_{i}$ is positive and that since $\sum_{i=1}^{k} a_{i} \leq r$ we can prove again that $\sum_{i=1}^{k} a_{i}^{2} \leq$ $(r-k+1)^{2}+(k-1)$, we get

$$
\sum_{i=1}^{k} \pi_{D_{i}}-k \leq \frac{H^{2}}{2}\left((r-k+1)^{2}+(k-1)\right)-\frac{3 H^{2}}{2} k .
$$

Some easy calculation shows that this is equivalent to our second statement.

Theorem 4.3. Let $X$ be a smooth projective surface of arithmetic Picard number one. Let $H$ be the ample generator of $\mathrm{NS}(X)$ and $S$ a finite set of rational points avoiding $H$. For any positive integer $r$, the minimum distance $d(X, r H, S)$ of the code $\mathcal{C}(X, r H, S)$ satisfies:

(i) if $3 H^{2}+H \cdot K_{X} \geq 0$, then

$$
d(X, r H, S) \geq\left\{\begin{array}{l}
\sharp S-\left(q+1+m \pi_{r H}\right) \text { if } r>2(q+1+m) / m H^{2}, \\
\sharp S-r\left(q+1+m \pi_{H}\right) \text { otherwise. }
\end{array}\right.
$$

(ii) If $3 H^{2}+H \cdot K_{X}<0$, then

$d(X, r H, S) \geq\left\{\begin{array}{l}\sharp S-(q+1+m)-m H^{2}\left(r^{2}-3\right) / 2 \text { if } r>2(q+1+m) / m H^{2}-3, \\ \sharp S-r\left(q+1+m-m H^{2}\right) \text { otherwise. }\end{array}\right.$

Proof. For any non-zero $f \in L(r H)$, we have by (9) and by point (1) of Theorem 2.3 the following inequality

$$
N(f) \leq k(q+1)+m \sum_{i=1}^{k} \pi_{D_{i}} .
$$


We apply Lemma 4.1 to bound $\sum_{i=1}^{k} \pi_{D_{i}}$. We get in the first case $N(f) \leq \phi(k)$ where $\phi(k):=m \pi_{(r-k+1) H}+k\left(q+1+m \pi_{H}\right)-m \pi_{H}$. Remark that $\pi_{(r-k+1) H}$ is quadratic in $k$ and so $\phi(k)$ is a quadratic function with positive leading coefficient. In [20, Lemma 2.2] Voloch and Zarzar proved that if $X$ has arithmetic Picard number one then $k \leq r$. Thus $\phi(k)$ attends its maximum for $k=1$ or for $k=r$ and $N(f) \leq \max \{\phi(1), \phi(r)\}$. A simple calculus shows that $\phi(1)-\phi(r)>$ 0 if and only if $r>2(q+1+m) / m H^{2}$. Since we have $d(X, r H, S) \geq \sharp S-$ $\max _{f \in L(r H) \backslash\{0\}} N(f)$, part (i) of the theorem is proved.

The treatment of part (ii) is the same, except that we use Lemma 4.1 to bound $\sum_{i=1}^{k} \pi_{D_{i}}$.

Remark 4.4. Little and Schenck have given bounds in $[14, \S 3]$ for the minimum distance of codes defined over algebraic surfaces of Picard number one. In particular, they obtain (if we keep the notations of Theorem 4.3): $d(X, r H, S) \geq$ $\sharp S-\left(q+1+m \pi_{H}\right)$ for $r=1\left(\left[14\right.\right.$, Th. 3.3]) and $d(X, r H, S) \geq \sharp S-r\left(q+1+m \pi_{H}\right)$ for $r>1$ and $q$ large ([14, Th. 3.5]). Comparing their bounds with Theorem 4.3, one can see that when $3 H^{2}+H \cdot K_{X} \geq 0$ we get the same bound for $r=1$ and also for $r>1$ without any hypothesis on $q$. Moreover, when $3 H^{2}+H . K_{X}<0$, our bounds improve the ones given by Little and Schenck, again without assuming large enough $q$ when $r>1$.

4.2. Surfaces without irreducible curves defined over $\mathbb{F}_{q}$ with small selfintersection and whose canonical divisor is either nef or anti-nef. We consider in this section surfaces $X$ such that there exists some integer $\beta \geq 0$ for which any $\mathbb{F}_{q}$-irreducible curve $D$ lying on $X$ and defined over $\mathbb{F}_{q}$ has selfintersection $D^{2} \geq \beta$. We prove in this case Lemma 4.5 below, from which we can tackle assumption (1) in Lemma 2.1 in case $\beta>0$. Unfortunately, Lemma 4.5 enables to fulfill assumption (2) of Lemma 2.1 only in case the intersection of the canonical divisor with $\mathbb{F}_{q}$-irreducible curves has constant sign, that is for surfaces of Section 3.1. The lower bound for the minimum distance we get is better than the one given in Theorem 3.2.

Let us propose some examples of surfaces with this property:

- simple abelian surfaces satisfy this property for $\beta=2$.

- Surfaces whose arithmetic Picard number is one. Indeed consider a curve $D$ defined over $\mathbb{F}_{q}$ on $X$, and assume that $\mathrm{NS}(X)=\mathbb{Z} H$ with $H$ ample. Then we have that $D=a H$ for some integer $a$. Since $H$ is ample we get $1 \leq D \cdot H=a H^{2}$ hence $a \geq 1$ and $D^{2}=a^{2} H^{2} \geq H^{2}$.

- Surfaces whose canonical divisor is anti-nef and without irreducible curves of arithmetic genus less or equal to $\ell>0$. Indeed the adjunction formula gives $D^{2}=2 \pi_{D}-2-D \cdot K_{X} \geq 2 \pi_{D}-2 \geq 2 \ell$.

Lemma 4.5. Let $X$ be a surface on which any $\mathbb{F}_{q}$-irreducible curve has selfintersection at least $\beta \geq 0$. Assume that $H$ is a rational effective ample divisor 
on $X$ and let $r$ be a positive integer. Let $D=\sum_{i=1}^{k} n_{i} D_{i}$ be the decomposition as a sum of $\mathbb{F}_{q}$-irreducible and reduced curves of an effective divisor $D$ linearly equivalent to $r H$. Then we have

(1) if $\beta>0$ then $k \leq r \sqrt{\frac{H^{2}}{\beta}}$;

(2) $\sum_{i=1}^{k}\left(2 \pi_{D_{i}}-2-D_{i} \cdot K_{X}\right) \leq \varphi(k)$, with

$$
\varphi(k):=(k-1) \beta+\left(r \sqrt{H^{2}}-(k-1) \sqrt{\beta}\right)^{2} .
$$

Proof. Since by hypothesis we have $\sqrt{\beta} \leq \sqrt{D_{i}^{2}}$, we deduce that $k \sqrt{\beta} \leq \sum_{i=1}^{k} n_{i} \sqrt{D_{i}^{2}}$. By (2), we get $k \sqrt{\beta} \leq \sum_{i=1}^{k} n_{i} \frac{D_{i} \cdot H}{\sqrt{H^{2}}}=\frac{r H \cdot H}{\sqrt{H^{2}}}=r \sqrt{H^{2}}$, from which the first item follows.

Setting $x_{i}:=\sqrt{2 \pi_{D_{i}}-2-D_{i} \cdot K_{X}}$, we have by adjunction formula $x_{i}=\sqrt{D_{i}^{2}} \geq$ $\sqrt{\beta}$. Moreover the previous inequalities ensure that $\sum_{i=1}^{k} x_{i} \leq \sum_{i=1}^{k} n_{i} \sqrt{D_{i}^{2}} \leq$ $r \sqrt{H^{2}}$. Then, the maximum of $\sum_{i=1}^{k}\left(2 \pi_{D_{i}}-2-D_{i} \cdot K_{X}\right)=\sum_{i=1}^{k} x_{i}^{2}$ under the conditions $x_{i} \geq \sqrt{\beta}$ and $\sum_{i=1}^{k} x_{i} \leq r \sqrt{H^{2}}$ is reached when each but one $x_{i}$ equals the minimum $\sqrt{\beta}$, and only one is equal to $r \sqrt{H^{2}}-(k-1) \sqrt{\beta}$, and this concludes the proof.

Theorem 4.6. Let $X$ be a surface on which any $\mathbb{F}_{q}$-irreducible curve has selfintersection at least $\beta>0$. Consider a rational effective ample divisor $H$ on $X$ avoiding a set $S$ of rational points and let $r$ be a positive integer. Then

$$
d(X, r H, S) \geq\left\{\begin{array}{l}
\sharp S-\max \left\{\psi(1), \psi\left(r \sqrt{\frac{H^{2}}{\beta}}\right)\right\}-\frac{m}{2} r \sqrt{\frac{H^{2}}{2 \beta}} \text { if } K_{X} \text { is nef, } \\
\sharp S-\max \left\{\psi(1), \psi\left(r \sqrt{\frac{H^{2}}{\beta}}\right)\right\} \text { if }-K_{X} \text { is nef }
\end{array}\right.
$$

with

$$
\psi(k):=\frac{m}{2} \varphi(k)+k(q+1+m),
$$

where $\varphi(k)$ is given by equation (13).

Proof. For any non-zero $f \in L(r H)$, we have by (9) and by point (1) of Theorem 2.3 that $N(f) \leq k(q+1)+m \sum_{i=1}^{k} \pi_{D_{i}}$. Lemma 4.5 implies that $N(f) \leq k(q+$ $1)+\frac{m}{2}\left(2 k+\varphi(k)+\sum_{i=1}^{k} D_{i} \cdot K_{X}\right)$. In case $K_{X}$ is nef, we have $\sum_{i=1}^{k} D_{i} \cdot K_{X} \leq$ $\sum_{i=1}^{k} n_{i} D_{i} \cdot K_{X}=r H . K_{X}$, and in case $-K_{X}$ is nef, we get $\sum_{i=1}^{k} D_{i} \cdot K_{X} \leq 0$, and the theorem follows.

4.3. Fibered surfaces with nef canonical divisor. We consider in this subsection AG codes from fibered surfaces whose canonical divisor is nef. We adopt the vocabulary of $[18$, III, $\S 8]$ and we refer the reader to this text for the basic notions we recall here. A fibered surface is a surjective morphism $\pi: X \rightarrow B$ from a smooth projective surface $X$ to a smooth absolutely irreducible curve $B$. We denote by $\pi_{0}$ the common arithmetic genus of the fibers and by $g_{B}$ the genus of the base curve $B$. Elliptic surfaces are among the first non-trivial examples of 
fibered surfaces. For such surfaces we have $\pi_{0}=1$ and the canonical divisor is always nef (see [6]).

We recall that on a fibered surface every divisor can be uniquely written as a sum of horizontal curves (that is mapped onto $B$ by $\pi$ ) and fibral curves (that is mapped onto a point by $\pi$ ).

Lemma 4.7. Let $\pi: X \rightarrow B$ be a fibered surface. Let $H$ be a rational effective ample divisor on $X$ and let $r$ be a positive integer. For any effective divisor $D$ linearly equivalent to $r H$, consider its decomposition $D=\sum_{i=1}^{k} n_{i} D_{i}$ as a sum of reduced $\mathbb{F}_{q}$-irreducible curves as in (8). Denote by $\bar{r}_{i}$ the number of absolutely irreducible components of $D_{i}$. Then, we have

$$
\sum_{i=1}^{k} \bar{r}_{i} \leq r H^{2}
$$

Proof. Write $D=\sum_{i=1}^{k} n_{i} D_{i}=\sum_{i=1}^{k} n_{i} \sum_{j=1}^{\bar{r}_{i}} D_{i, j}$ where the $D_{i, j}$ are the absolutely irreducible components of $D_{i}$.

We use that $n_{i}>0$, that $D$ is numerically equivalent to $r H$ and that $D_{i, j} . H>0$ to get

$$
\sum_{i=1}^{k} \bar{r}_{i} \leq \sum_{i=1}^{k} \sum_{j=1}^{\bar{r}_{i}} D_{i, j} \cdot H \leq \sum_{i=1}^{k} n_{i} \sum_{j=1}^{\bar{r}_{i}} D_{i, j} . H=\sum_{i=1}^{k} n_{i} D_{i} . H=r H . H,
$$

which proves the lemma.

The next theorem involves the defect $\delta(B)$ of a smooth absolutely irreducible curve $B$ defined over $\mathbb{F}_{q}$ of genus $g_{B}$, which is defined by

$$
\delta(B):=q+1+m g_{B}-\sharp B\left(\mathbb{F}_{q}\right) .
$$

By the Serre-Weil theorem this defect is a non-negative number. The so-called maximal curves have defect 0 , and the smaller the number of rational points on $B$ is, the greater the defect is.

Theorem 4.8. Let $\pi: X \rightarrow B$ be a fibered surface whose canonical divisor $K_{X}$ is nef. Assume that $H$ is a rational effective ample divisor on $X$ having at least one horizontal component and avoiding a set $S$ of rational points. For any positive integer $r$ the minimum distance of $\mathcal{C}(X, r H, S)$ satisfies

$$
d(X, r H, S) \geq d^{*}(X, r H, S)+\delta(B)
$$

where $d^{*}(X, r H, S)$ is given by formula (12).

Recall that the general bound we obtain in Theorem 3.2 in Section 3 for surfaces with nef canonical divisor is $d(X, r H, S) \geq d^{*}(X, r H, S)$, thus the bound from Theorem 4.8 is always equal or better. Actually Theorem 4.8 is surprising, since it says that the lower bound for the minimum distance is all the more large because the defect $\delta(B)$ is. Consequently it looks like considering fibered surfaces on 
curves with few rational points and large genus could lead to potentially good codes.

Proof. Recall that for any non-zero $f \in L(r H)$, we have $d(X, r H, S) \geq \sharp S-N(f)$, and that $N(f) \leq \sum_{i=1}^{k} \sharp D_{i}\left(\mathbb{F}_{q}\right)$ if we use the notation $D_{f}:=r H+(f)=$ $\sum_{i=1}^{k} n_{i} D_{i}$ introduced in (8). We again denote by $\bar{r}_{i}$ the number of absolutely irreducible components of $D_{i}$. In order to introduce the $\mathbb{F}_{q}$-irreducible components of $D_{f}$, write $k=h+v$, where $h$ (respectively $v$ ) is the number of horizontal curves denoted by $H_{1}, \ldots, H_{h}$, (respectively fibral curves denoted by $F_{1}, \ldots, F_{v}$ ). Then we get $N(f) \leq \sum_{i=1}^{h} \sharp H_{i}\left(\mathbb{F}_{q}\right)+\sum_{i=1}^{v} \sharp F_{i}\left(\mathbb{F}_{q}\right)$. Since $B$ is a smooth curve, the morphisms $H_{i} \rightarrow B$ are flat. Now applying Proposition 2.4 to horizontal curves and Theorem 2.3 to fibral curves gives

$$
\begin{aligned}
N(f) & \leq h\left(\sharp B\left(\mathbb{F}_{q}\right)-m g_{B}\right)+m \sum_{i=1}^{h} \pi_{H_{i}}+q \sum_{i=1}^{h}\left(\bar{r}_{i}-1\right)+q v+v+m \sum_{i=1}^{v} \pi_{F_{i}} \\
& =h\left(\sharp B\left(\mathbb{F}_{q}\right)-m g_{B}-q\right)+m \sum_{i=1}^{k} \pi_{D_{i}}+q \sum_{i=1}^{k} \bar{r}_{i}+v,
\end{aligned}
$$

where we used the fact that $v \leq \sum_{i=h+1}^{k} \bar{r}_{i}$.

Since the canonical divisor of the fibered surface is assumed to be nef, Lemma 3.1 gives a bound for $\sum_{i=1}^{k} \pi_{D_{i}}$. We set $v=k-h$ and we use Lemma 4.7 with (14) to obtain

$$
\begin{aligned}
N(f) & \leq h\left(\sharp B\left(\mathbb{F}_{q}\right)-m g_{B}-q\right)+m\left(\pi_{r H}-1\right)+m k+q r H^{2}+v \\
& =h\left(\sharp B\left(\mathbb{F}_{q}\right)-m g_{B}-q-1\right)+m\left(\pi_{r H}-1\right)+m k+q r H^{2}+k \\
& =-h \delta(B)+m\left(\pi_{r H}-1\right)+m k+q r H^{2}+k .
\end{aligned}
$$

Now, $D_{f} . F \equiv r H . F>0$ since $F$ is a generic fiber and $r H$ is assumed to have at least one horizontal component. Thus, $D_{f}$ has also at least one horizontal component, that is $h \geq 1$. Moreover, again from Lemma 3.1 we have $k \leq r H^{2}$. As the defect $\delta(B)$ is non-negative it follows that

$$
N(f) \leq-\delta(B)+r H^{2}(q+1+m)+m\left(\pi_{r H}-1\right)
$$

and the theorem is proved.

4.4. Fibered surfaces whose singular fibers are irreducible. In this subsection we drop off the condition on the canonical divisor. Instead, we assume that every singular fiber on $X$ is $\mathbb{F}_{q}$-irreducible. To construct examples of such surfaces, fix any $d \geq 1$ and recall that the dimension of the space of degree $d$ homogeneous polynomials in three variables is $\left(\begin{array}{c}d+2 \\ 2\end{array}\right)$. Hence the space $\mathcal{P}_{d}$ of plane curves of degree $d$ is $\mathcal{P}_{d}=\mathbb{P}^{\left(\begin{array}{c}d+2 \\ 2\end{array}\right)-1}$. Any curve $B$ drawn in $\mathcal{P}_{d}$ gives rise to a fibered 
surface, whose fibers are plane curves of degree $d$, that is with $\pi_{0}=\frac{(d-1)(d-2)}{2}$. The locus of singular curves being a subvariety of $\mathcal{P}_{d}$, choosing $B$ not contained in this singular locus yields to a fibered surface with smooth generic fiber. As the locus of reducible curves has high codimension in $\mathcal{P}_{d}$, choosing $B$ avoiding this locus yields to fibered surfaces without reducible fibers.

We consider the case where $\pi_{0}$ and $g_{B}$ are both at least 2 and we set $\ell=$ $\min \left(\pi_{0}, g_{B}\right)-1 \geq 1$. We recall again that every divisor on $X$ can be uniquely written as a sum of horizontal and fibral curves. If we denote by $H$ an horizontal curve and by $V$ a fibral curve defined over $\mathbb{F}_{q}$, we have that $\pi_{H} \geq g_{B}$ and $\pi_{V}=\pi_{0}$. Therefore, in this setting, $X$ contains no $\mathbb{F}_{q}$-irreducible curves defined over $\mathbb{F}_{q}$ of arithmetic genus smaller than or equal to $\ell$. Thus Lemma 3.3 applies and gives the same bound for $\sum_{i=1}^{k} \pi_{i}$ as when $K_{X}$ is nef and the bound $k \leq\left(\pi_{r H}-1\right) / \ell$ for the number $k$ of $\mathbb{F}_{q}$-irreducible components of $D_{f}$. We consider this new bound for $k$ in the proof of Theorem 4.8 and we get instead the following result.

Theorem 4.9. Let $\pi: X \rightarrow B$ be a fibered surface. We consider a rational effective ample divisor $H$ on $X$ having at least one horizontal component and avoiding a set $S$ of rational points. Let $r$ be a positive integer. We denote by $g_{B}$ the genus of $B$ and by $\pi_{0}$ the arithmetic genus of the fibers and we set $\ell=$ $\min \left(\pi_{0}, g_{B}\right)-1$. Suppose that every singular fiber is $\mathbb{F}_{q}$-irreducible and that $\ell \geq 1$. Then the minimum distance of $\mathcal{C}(X, r H, S)$ satisfies

$$
d(X, r H, S) \geq d^{*}(X, r H, S)+\left(r H^{2}-\frac{\pi_{r H}-1}{\ell}\right)(q+1+m)+\delta(B),
$$

where $d^{*}(X, r H, S)$ is given by formula (12).

Naturally this bound is better than the one in Theorem 4.8 if and only if $\pi_{r H}-1<\ell r H^{2}$. Furthermore it improves the bound of Theorem 3.4 by the addition of the non-negative term $\delta(B)$.

\section{AN EXAMPLE: SURFACES IN $\mathbb{P}^{3}$}

This section is devoted to the study of the minimum distance of AG codes over a surface $X$ of degree $d \geq 3$ embedded in $\mathbb{P}^{3}$.

We consider the class $L$ of an hyperplane section of $X$. So $L$ is ample, $L^{2}=d$ and the canonical divisor on $X$ is $K_{X}=(d-4) L$ (see [17, p.212]). In this setting, we fix a rational effective ample divisor $H$ and $r$ a positive integer. We apply our former theorems to this context to give bounds on the minimum distance of the code $\mathcal{C}(X, r H, S)$.

We recall that cubic surfaces are considered by Voloch and Zarzar in [20] and [21] to provide computationally good codes. In Section 4 of [14] Little and Schenck propose theoretical and experimental results for surfaces in $\mathbb{P}^{3}$ always in the prospect of finding good codes. We also contribute to this study with a view to bounding the minimum distance according to the geometry of the surface. 
Proposition 5.1. Let $X$ be a surface of degree $d \geq 3$ embedded in $\mathbb{P}^{3}$. Consider a rational effective ample divisor $H$ avoiding a set $S$ of rational points on $X$ and let $r$ be a positive integer. Then the minimum distance of the code $\mathcal{C}(X, r H, S)$ satisfies

(1) if $X$ is a cubic surface, then

$$
d(X, r H, S) \geq d^{*}(X, r H, S)+m r\left(\pi_{H}-1\right) .
$$

(2) If $X$ has degree $d \geq 4$ then

$$
d(X, r H, S) \geq d^{*}(X, r H, S)
$$

where

$$
d^{*}(X, r H, S)=\sharp S-r H^{2}(q+1+m)-m\left(\pi_{r H}-1\right)
$$

is the function defined in (12).

Proof. Since $K_{X}=(d-4) L$ we have for cubic surfaces that $K_{X}=-L$ and thus the canonical divisor is anti-ample, while for surfaces of degree $d \geq 4$ the canonical divisor is ample or the zero divisor, thus is nef. Hence we can apply Theorem 3.2 from which the proposition follows.

5.1. Surfaces in $\mathbb{P}^{3}$ without irreducible curves of low genus. In the complex setting, the Noether-Lefschetz theorem asserts that a general surface $X$ of degree $d \geq 4$ in $\mathbb{P}^{3}$ is such that $\operatorname{Pic}(X)=\mathbb{Z} L$, where $L$ is the class of an hyperplane section (see [9]). Here, general means outside a countable union of proper subvarieties of the projective space parametrizing the surfaces of degree $d$ in $\mathbb{P}^{3}$. Even if we do not know an analog of this statement in our context, it suggests us the strong assumptions we take in this subsection, namely in Lemma 5.2 and Proposition 5.3.

Lemma 5.2. Let $X$ be a surface of degree $d \geq 4$ in $\mathbb{P}^{3}$ of arithmetic Picard number one. Suppose that $\mathrm{NS}(X)$ is generated by the class of an hyperplane section L. Consider an $\mathbb{F}_{q}$-irreducible curve $D$ on $X$ of arithmetic genus $\pi_{D}$. Then

$$
\pi_{D} \geq(d-1)(d-2) / 2 .
$$

Proof. Let $a$ be the integer such that $D=a L$ in $\operatorname{NS}(X)$. Since $D$ is an $\mathbb{F}_{q^{-}}$ irreducible curve and $L$ is ample, we must have $a>0$. Then, using the adjonction formula, we get

$$
\begin{aligned}
2 \pi_{D}-2 & =D^{2}+D \cdot K=a^{2} L^{2}+a L \cdot(d-4) L \\
& =a^{2} d+a d(d-4) \geq d+d(d-4),
\end{aligned}
$$

and thus $\pi_{D} \geq(d-1)(d-2) / 2$.

By the previous lemma it is straightforward that in our context $X$ does not contain any $\mathbb{F}_{q}$-irreducible curves of arithmetic genus smaller than or equal to $\ell$ for $\ell=(d-1)(d-2) / 2-1=d(d-3) / 2$. This allows us to apply Theorem 3.4, and get the following proposition. 
Proposition 5.3. Let $X$ be a degree $d \geq 4$ surface in $\mathbb{P}^{3}$ of arithmetic Picard number one whose Néron-Severi group $\mathrm{NS}(X)$ is generated by the class of an hyperplane section L. Assume that $S$ is a set of rational points avoiding L. For any positive integer $r$ the minimum distance of the code $\mathcal{C}(X, r L, S)$ satisfies

$$
d(X, r L, S) \geq d^{*}(X, r L, S, L)+r d\left(1-\frac{r+d-4}{d(d-3)}\right)(q+1+m)
$$

where

$$
d^{*}(X, r L, S, L)=\sharp S-r d(q+1+m)-\operatorname{mrd}(r+d-4) / 2 .
$$

5.2. Surfaces in $\mathbb{P}^{3}$ of arithmetic Picard number one. In this subsection we suppose that the arithmetic Picard number of $X$ is one, but we do not take the assumption that the Néron-Severi group is generated by an hyperplane section. Also in this case we can apply Theorem 4.3 which brings us to the following proposition.

Proposition 5.4. Let $X$ be a surface of degree $d \geq 4$ in $\mathbb{P}^{3}$. Assume that $\mathrm{NS}(X)=\mathbb{Z} H$ for an ample divisor $H$. Consider $L=h H$, the class of an hyperplane section of $X$, for $h$ a positive integer. Let $S$ be a set of rational points on $X$ avoiding $H$ and let $r$ be a positive integer. Then the minimum distance of the code $\mathcal{C}(X, r H, S)$ satisfies

$d(X, r H, S) \geq\left\{\begin{array}{l}\sharp S-(q+1+m)-r H^{2}(r+h(d-4)) / 2 \text { if } r>2(q+1+m) / m H^{2}, \\ \sharp S-r(q+1+m)-r H^{2}(1+h(d-4)) / 2 \text { otherwise. }\end{array}\right.$

Proof. Since we have $3 H^{2}+H . K_{X}=3 H^{2}+H .(d-4) L=3 H^{2}+h(d-4) H^{2}=$ $H^{2}(3+h(d-4)) \geq 0$, we can apply point (i) of Theorem 4.3 from which the proposition follows.

Acknowledgments: The authors would like to thank the anonymous referee for relevant observations.

\section{REFERENCES}

[1] Y. Aubry. "Algebraic geometric codes on surfaces". talk at Eurocode'92 International symposium on coding theory and applications (1992, Udine, Italie), in Ph.D. thesis of the University of Aix-Marseille II, France (1993), hal-00979000. URL: https://hal archives-ouvertes.fr/hal-00979000/file/EuroCode.19

[2] Y. Aubry and M. Perret. "A Weil theorem for singular curves". In: Arithmetic, geometry and coding theory (Luminy, 1993). de Gruyter, Berlin, 1996, pp. 1-7.

[3] Y. Aubry and M. Perret. "On the characteristic polynomials of the Frobenius endomorphism for projective curves over finite fields". In: Finite Fields Appl. 10.3 (2004), pp. 412-431. ISSN: 1071-5797. DOI: 10.1016/j.ffa.2003.09.005. URL: https://doi.org/10.1016/j.ffa.2003.09.005. 
[4] Y. Aubry et al. "Algebraic geometry codes over abelian surfaces containing no absolutely irreducible curves of low genus". In: CoRR abs/1904.08227 (2019). arXiv: 1904.08227. URL: http://arxiv.org/abs/1904.08227.

[5] R. Blache et al. "Anticanonical codes from del Pezzo surfaces with Picard rank one". In: CoRR abs/1903.09397 (2019). arXiv: 1903.09397. URL: http://arxiv.org/abs/1903.09397.

[6] E. Bombieri and D. B. Mumford. "Enriques' classification of surfaces in char. p, III". In: Inventiones Mathematicae 35.1 (1976), pp. 197-232.

[7] A. Couvreur. "Construction of rational surfaces yielding good codes". In: $F i$ nite Fields Appl. 17.5 (2011), pp. 424-441. ISSN: 1071-5797. DOI: 10.1016/j .ffa. 2011.02 .00 URL: https://doi.org/10.1016/j.ffa.2011.02.007.

[8] V. D. Goppa. "Codes on algebraic curves". In: Dokl. Akad. Nauk SSSR 259.6 (1981), pp. 1289-1290. ISSN: 0002-3264.

[9] P. Griffiths and J. Harris. "On the Noether-Lefschetz theorem and some remarks on codimension-two cycles". In: Mathematische Annalen 271.1 (1985), pp. 31-51.

[10] S. Haloui. "Codes from Jacobian surfaces". In: Arithmetic, geometry, cryptography and coding theory. Vol. 686. Contemp. Math. Amer. Math. Soc., Providence, RI, 2017, pp. 123-135.

[11] J. P. Hansen. "Toric surfaces and error-correcting codes". In: Coding theory, Cryptography and related areas. Springer, 2000, pp. 132-142.

[12] S. H. Hansen. "Error-correcting codes from higher-dimensional varieties". In: Finite Fields Appl. 7.4 (2001), pp. 531-552. ISSN: 1071-5797.

[13] R. Hartshorne. Algebraic Geometry. Graduate Texts in Mathematics, No. 52. Springer-Verlag, New York-Heidelberg, 1977.

[14] J. Little and H. Schenck. "Codes from surfaces with small Picard number". In: SIAM J. Appl. Algebra Geom. 2.2 (2018), pp. 242-258. ISSN: 2470-6566. DOI: 10.1137/17M1128277. URL: https://doi.org/10.1137/17M1128277.

[15] J. B. Little. "Algebraic geometry codes from higher dimensional varieties". In: Advances in algebraic geometry codes. Vol. 5. Ser. Coding Theory Cryptol. World Sci. Publ., Hackensack, NJ, 2008, pp. 257-293.

[16] J. Nardi. "Algebraic geometric codes on minimal Hirzebruch surfaces". In: $J$. Algebra 535 (2019), pp. 556-597. ISSN: 0021-8693. DOI: 10.1016/j . jalgebra. 2019.06.022. URL: https://doi.org/10.1016/j.jalgebra.2019.06.022.

[17] I. R. Shafarevich. Basic algebraic geometry. 1. Second. Varieties in projective space, Translated from the 1988 Russian edition and with notes by Miles Reid. Springer-Verlag, Berlin, 1994, pp. xx+303. ISBN: 3-540-54812-2.

[18] J. H. Silverman. Advanced Topics in the Arithmetic of Elliptic Curves. Graduate Texts in Mathematics, No. 151. Springer-Verlag, New York-Heidelberg, 1995.

[19] M. A. Tsfasman, S. G. Vlăduţ, and T. Zink. "Modular curves, Shimura curves, and Goppa codes, better than Varshamov-Gilbert bound". In: Math. 
Nachr. 109 (1982), pp. 21-28. ISSN: 0025-584X. DOI: 10.1002/mana. 19821090103. URL: https://doi.org/10.1002/mana.19821090103.

[20] J. F. Voloch and M. Zarzar. "Algebraic geometric codes on surfaces". In: Arithmetics, geometry, and coding theory (AGCT 2005). Vol. 21. Sémin. Congr. Soc. Math. France, Paris, 2010, pp. 211-216.

[21] M. Zarzar. "Error-correcting codes on low rank surfaces". In: Finite Fields Appl. 13.4 (2007), pp. 727-737. ISSN: 1071-5797. DOI: 10.1016/j.ffa.2007.05.001. URL: https://doi.org/10.1016/j.ffa.2007.05.001.

Yves Aubry, Institut de Mathématiques de Toulon - IMATH, Université de Toulon and Institut de Mathématiques de Marseille - I2M, Aix Marseille Université, CNRS, Centrale Marseille, UMR 7373, France E-mail address: yves.aubry@univ-tln.fr

Elena Berardini, Institut de Mathématiques de Marseille - I2M, Aix Marseille Université, CNRS, Centrale Marseille, UMR 7373, France E-mail address: elena_berardini@hotmail.it

Fabien Herbaut, INSPE Nice-Toulon, Université Côte D’Azur, Institut de Mathématiques de Toulon - IMATH, Université de Toulon, France E-mail address: fabien.herbaut@univ-cotedazur.fr

Marc Perret, Institut de Mathématiques de Toulouse, UMR 5219, Université de Toulouse, CNRS, UT2J, F-31058 Toulouse, France E-mail address: perret@univ-tlse2.fr 